\title{
SEARCHING FOR DARK MATTER- THEORETICAL RATES AND EXCLUSION PLOTS DUE TO THE SPIN
}

\author{
J. D. Vergados \\ Theoretical Physics Division, University of Ioannina, \\ Ioannina, Gr 451 10, Greece \\ and \\ University of Tuebingen, Tuebingen, Germany.
}

(Dated: May 28, 2018)

\begin{abstract}
The recent WMAP data have confirmed that exotic dark matter together with the vacuum energy (cosmological constant) dominate in the flat Universe. The nature of the dark matter constituents cannot be determined till they are directly detected. Recent developments in particle physics provide a number of candidates as constituents of dark matter, called Weakly Interacting Massive Particles (WIMPs). Since these interact weakly and are of low energy, they cannot excite the target and can only be detected via measuring the recoiling nucleus. For all WIMPs, including the most popular candidate, the lightest supersymmetric particle (LSP), the relevant cross sections arise out of the following mechanisms: i) The coherent mode, due to the scalar interaction. ii) The charge coherent mode, with only proton contribution, as in the recent case of secluded dark matter scenario and iii) The spin contribution arising from the axial current. In this paper we will focus on the spin contribution, which maybe important, especially for light targets.
\end{abstract}

PACS numbers: $95.35 .+\mathrm{d}, 12.60 . \mathrm{Jv}$

\section{INTRODUCTION}

Combining the recent WMAP data 1] with other experiments one finds that most of the matter in the Universe is cold dark matter (CDM):

$$
\Omega_{b}=0.0456 \pm 0.0015, \Omega_{C D M}=0.228 \pm 0.013, \Omega_{\Lambda}=0.726 \pm 0.015
$$

Since the non exotic component cannot exceed $40 \%$ of the CDM [2], there is room for exotic WIMPs (Weakly Interacting Massive Particles). In fact the DAMA experiment [3] has claimed the observation of one signal in direct detection of a WIMP, which with better statistics has subsequently been interpreted as a modulation signal [4]. These data, however, if they are due to the coherent process, are not consistent with other recent experiments, see e.g. EDELWEISS [5], CDMS [6] and XENON [7]. The DAMA results could still be interpreted as due to the spin cross section, but with a new interpretation of the extracted nucleon cross section.

Supersymmetry naturally provides candidates for the dark matter constituents (see, e.g, the review [8]). In the most favored scenario of supersymmetry the LSP (Lightest Supersymmetric Particle) can be simply described as a Majorana fermion, a linear combination of the neutral components of the gauginos and higgsinos [8], [9]. In most calculations the neutralino is assumed to be primarily a gaugino, usually a bino. Models which predict a substantial fraction of higgsino lead to a relatively large spin induced cross section due to the Z-exchange. Such models have been less popular, since they tend to violate the relic abundance constraint. These fairly stringent constrains, however, apply only in the thermal production mechanism. Furthermore they do not affect the WIMP density in our vicinity derived from the rotational curves. Thus one may assume that large spin cross sections are possible in such models [10], 11], which are non-universal gaugino mass models and give rise to large higgsino components. Sizable spin cross sections also arise in the context of other models, which have appeared recently [12], 13]-[14] (see also Ellis et al [15] for an update).

Spin induced cross sections also arize in the case Kaluza-Klein (K-K) WIMPs in models with Universal Extra Dimensions (UED) 16. This occurs regardless of whether the WIMP is a K-K boson or a K-K neutrino. They can also arise in technicolor theories [17]. In the Ultra Minimal Walking Technicolor model [18, 19, 20] there exist singlet composite Majorana fermionic states. These, taken as dark matter candidates, can lead to spin induced cross sections.

Knowledge of the spin induced nucleon cross section is very important since, for some special targets, it may lead to transitions to excited nuclear states, which provide the attractive signature of detecting the de-excitation $\gamma$ rays in or without coincidence with the recoiling nucleus 21, 22]. Furthermore it may dominate in light systems like ${ }^{3} \mathrm{He}$ and ${ }^{19} \mathrm{~F}$, which offer some attractive advantages [23], [24].

In light of the above it is clear that the spin mechanism needs be considered. In this article we will discuss the theoretical ingredients needed to obtain the WIMP-nuclear spin induced cross sections. Then we will give expressions 
for and calculate the event rates, both modulated and unmodulated, in terms of the elementary proton $\left(\sigma_{p}\right)$ or neutron $\left(\sigma_{n}\right)$ cross sections. After that we will provide exlusion plots in the $\left(\sigma_{p}, \sigma_{n}\right)$ plane in terms of parameters relevant to the experiments, for various targets of experimental interest. Our results will be presented in a way that will make them useful in the analysis of the data of the odd mass targets.

\section{THE ESSENTIAL THEORETICAL INGREDIENTS OF DIRECT DETECTION.}

Even though there exists firm indirect evidence for a halo of dark matter in galaxies from the observed rotational curves, it is essential to directly detect such matter. Such a direct detection, among other things, may also unravel the nature of the constituent of cold dark matter, namely the Weakly Interacting Massive Particle (WIMP). The possibility of such detection, however, depends on the nature of its constituents. Our main conclusions apply to all heavy WIMPs. Since the WIMP is expected to be very massive, $m_{\chi} \geq 30 \mathrm{GeV}$, and extremely non relativistic with average kinetic energy $T \approx 50 \mathrm{KeV}\left(m_{\chi} / 100 \mathrm{GeV}\right)$, it can be directly detected mainly via the recoiling of a nucleus $(\mathrm{A}, \mathrm{Z})$ in elastic scattering. The event rate for such a process can be computed from the following ingredients:

1. An effective Lagrangian at the elementary particle (quark) level obtained in the framework of supersymmetry as described, e.g., in Refs [9, 25]. An analogous procedure can be found in the case of K-K WIMPs in Universal Extra Dimension (UED) models [16] and technicolor theories [17].

2. A well defined procedure for transforming the amplitude obtained using the above mentioned effective Lagrangian from the quark to the nucleon level where This step is not trivial, since the obtained results depend crucially on the content of the nucleon in quarks other than $\mathrm{u}$ and $\mathrm{d}$. This is particularly true for the scalar couplings, which are proportional to the quark masses [26, 26, 27, 28] as well as the isoscalar axial coupling.

3. Nuclear matrix elements.

These must be obtained with as reliable as possible many body nuclear wave functions. Fortunately in the most studied case of the scalar coupling the situation is quite simple, since then one needs only the nuclear form factor. Some progress has also been made in obtaining reliable static spin matrix elements and spin response functions [29, 30, 31, 32]

4. A velocity distribution for WIMPs

In this article we will follow the standard practice and assume a M-B distribution, but other perhaps more realistic velocity distributions have also recently been considered [33, 34]

Since the obtained rates are very low, one would like to be able to exploit the modulation of the event rates due to the earth's revolution around the sun [35, 36, 37, 38]

\section{THE WIMP NUCLEUS CROSS SECTIONS}

The standard (non directional) differential rate can be written as

$$
d R=\frac{\rho(0)}{m_{\chi^{0}}} \frac{m}{A m_{N}} d \sigma(u, v)|v|,
$$

where $\mathrm{m}$ is the detector mass, $\rho(0)=0.3 \mathrm{GeV} / \mathrm{cm}^{3}$ is the WIMP density in our vicinity, $v$ its velocity and $m_{\chi^{0}}$ its mass and $d \sigma(u, v)$ is given by

$$
d \sigma(u, v)==\frac{d u}{2\left(\mu_{r} b v\right)^{2}}\left[\bar{\Sigma}_{S} F^{2}(u)+\bar{\Sigma}_{\text {spin }} F_{11}(u)\right],
$$

where $u$ is the energy transfer $Q$ in dimensionless units given by

$$
u=\frac{Q}{Q_{0}}, Q_{0}=\left[m_{p} A b\right]^{-2}=40 A^{-4 / 3} \mathrm{MeV},
$$

with $b$ is the nuclear (harmonic oscillator) size parameter. $F(u)$ is the nuclear form factor and $F_{11}(u)$ is the spin response function associated with the isovector channel. The scalar cross section is given by:

$$
\bar{\Sigma}_{S} \approx \sigma_{N, \chi^{0}}^{S}\left(\frac{\mu_{r}}{\mu_{r}(p)}\right)^{2} A^{2}
$$




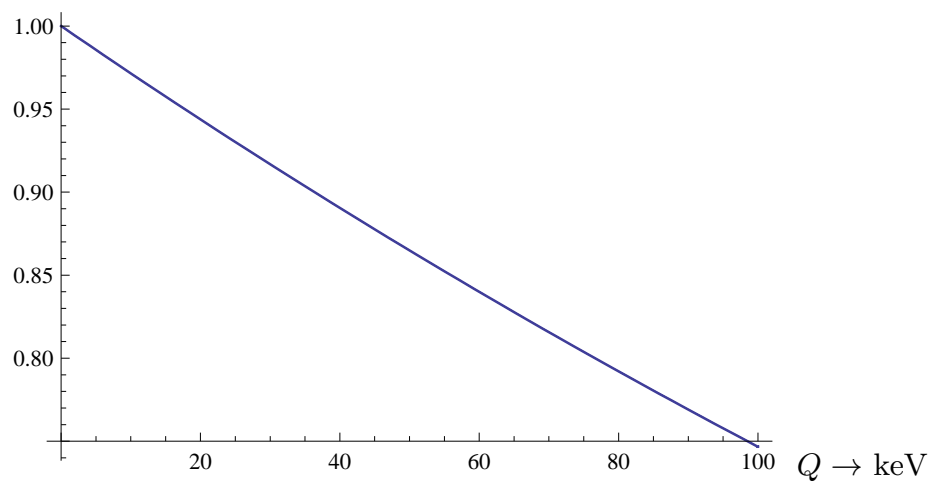

FIG. 1: The spin response functions $F_{11}(Q), F_{01}(Q)$ and $F_{00}(Q)$ in the case of the target ${ }^{19} \mathrm{~F}$ as a function of the energy transfer. In the region of interest for dark matter searches they are not distinguishable.

$\sigma_{N, \chi^{0}}^{S}$ is the WIMP-nucleon scalar cross section. Note that, since the heavy quarks dominate, the isovector contribution is negligible, i.e. the proton and nucleon cross sections are the same. The spin Cross section is given by:

$$
\begin{gathered}
\bar{\Sigma}_{\text {spin }}=\left(\frac{\mu_{r}}{\mu_{r}(p)}\right)^{2} \sigma_{p, \chi^{0}}^{\text {spin }} \zeta_{\text {spin }}, \zeta_{\text {spin }}=\frac{1}{3\left(1+\frac{f_{A}^{0}}{f_{A}^{1}}\right)^{2}} S(u), \\
\left.S(u)=\left[\left(\frac{f_{A}^{0}}{f_{A}^{1}} \Omega_{0}(0)\right)^{2} \frac{F_{00}(u)}{F_{11}(u)}+2 \frac{f_{A}^{0}}{f_{A}^{1}} \Omega_{0}(0) \Omega_{1}(0) \frac{F_{01}(u)}{F_{11}(u)}+\Omega_{1}(0)\right)^{2}\right] .
\end{gathered}
$$

The spin response functions $F_{i j}$, properly normalized to unity at momentum transfer zero, in the energy transfers of interest are almost the same for all isospin channels $i, j ; i, j=0,1$. As a matter of fact in the case of ${ }^{19} \mathrm{~F}$ we get:

$$
\begin{gathered}
F_{11}(u)=e^{-u}\left(0.0119 u^{4}-0.1450 u^{3}+0.6620 u^{2}-\frac{4 u}{3}+1\right), \\
F_{01}=e^{-u}\left(0.0124 u^{4}-0.1487 u^{3}+0.6677 u^{2}-\frac{4 u}{3}+1\right), \\
F_{00}=e^{-u}\left(0.0131 u^{4}-0.1525 u^{3}+0.6733 u^{2}-\frac{4 u}{3}+1\right)
\end{gathered}
$$

Thus they are indistinguishable (see Fig. 1) for the energy transfers of interest. Hence

$$
S(u) \approx S(0)=\left(\frac{f_{A}^{0}}{f_{A}^{1}} \Omega_{0}(0)+\Omega_{1}(0)\right)^{2} .
$$

The nuclear matrix elements $\Omega_{1}(0)\left(\Omega_{0}(0)\right)$ associated with the isovector (isoscalar) components are normalized so that, in the case of the proton, they yield $\zeta_{\text {spin }}=1$ at $u=0$.

The couplings $f_{A}^{1}\left(f_{A}^{0}\right)$ are obtained by multiplying the corresponding elementary amplitudes obtained at the quark level by suitable renormalization factors $g_{A}^{0}$ and $g_{A}^{1}$ given in terms of the quantities $\Delta q$ given by Ellis [39]

$$
g_{A}^{0}=\Delta u+\Delta d+\Delta s=0.77-0.49-0.15=0.13, g_{A}^{1}=\Delta u-\Delta d=1.26
$$

Thus, barring very unusual circumstances at the quark level, the isovector component is expected to be dominant. It is for this reason that we started our discussion in the isospin basis.

Heavy nuclei, however, are theoretically described in terms of protons and neutrons and the experiments are also analyzed this way. So will present our results in this basis. The proton and neutron cross section are given by:

$$
\sigma_{p, \chi^{0}}^{\text {spin }}=3 \sigma_{0}\left|f_{A}^{0}+f_{A}^{1}\right|^{2}=3 \sigma_{0}\left|a_{p}\right|^{2}, \sigma_{n, \chi^{0}}^{\text {spin }}=3 \sigma_{0}\left|f_{A}^{0}-f_{A}^{1}\right|^{2}=3 \sigma_{0}\left|a_{n}\right|^{2}
$$


TABLE I: The static spin matrix elements for various nuclei. For ${ }^{3} \mathrm{He}$ see Moulin, Mayet and Santos [41]. For the other light nuclei the calculations are from DIVARI 29]. For ${ }^{73} \mathrm{Ge}$ and ${ }^{127} \mathrm{I}$ the results presented are from Ressel et al [30] $\left(^{*}\right)$ and the Finish group et al [31] (**). For ${ }^{207} \mathrm{~Pb}$ they were obtained by the Ioannina team (+). [40], 32].

\begin{tabular}{lrrrrrrrr}
\hline \hline & & & & & & & & \\
& ${ }^{3} \mathrm{He}$ & ${ }^{19} \mathrm{~F}$ & ${ }^{29} \mathrm{Si}$ & ${ }^{23} \mathrm{Na}$ & ${ }^{73} \mathrm{Ge}$ & ${ }^{127} \mathrm{I}^{*}$ & ${ }^{127} \mathrm{I}^{* *}$ & ${ }^{207} \mathrm{~Pb}^{+}$ \\
\hline$\Omega_{0}(0)$ & 1.244 & 1.616 & 0.455 & 0.691 & 1.075 & 1.815 & 1.220 & 0.552 \\
$\Omega_{1}(0)$ & -1.527 & 1.675 & -0.461 & 0.588 & -1.003 & 1.105 & 1.230 & -0.480 \\
$\Omega_{p}(0)$ & -0.141 & 1.646 & -0.003 & 0.640 & 0.036 & 1.460 & 1.225 & 0.036 \\
$\Omega_{n}(0)$ & 1.386 & -0.030 & 0.459 & 0.051 & 1.040 & 0.355 & -0.005 & 0.516 \\
$\mu_{t h}$ & & 2.91 & -0.50 & 2.22 & & & & \\
$\mu_{\text {exp }}$ & & 2.62 & -0.56 & 2.22 & & & & \\
$\frac{\mu_{\text {th }(\text { spin })}}{\mu_{\text {exp }}}$ & 0.91 & 0.99 & 0.57 & & & & \\
\hline \hline
\end{tabular}

with $a_{p}$ and $a_{n}$ are the proton and neutron spin amplitudes, which, of course, depend on the model. In the case of the LSP 25]

$$
\sigma_{0}=\frac{1}{2 \pi}\left(G_{F} m_{p}\right)^{2}=0.77 \times 10^{-38} \mathrm{~cm}^{2}=0.77 \times 10^{-2} p b
$$

In extracting limits on the nucleon cross sections from the data we will find it convenient to write:

$$
\bar{\Sigma}_{\text {spin }}=\left(\frac{\mu_{r}}{\mu_{r}(p)}\right)^{2} \sigma_{\text {nuc }}^{\text {spin }}, \sigma_{\text {nuc }}^{\text {spin }}=\frac{1}{3}\left|\Omega_{p} \sqrt{\sigma_{p}}+\Omega_{n} \sqrt{\sigma_{n}} e^{i \delta}\right|^{2}=\frac{1}{3}|| \Omega_{p}\left|\sqrt{\sigma_{p}}+\right| \Omega_{n}\left|\sqrt{\sigma_{n}} e^{i\left(\delta+\delta_{A}\right)}\right|^{2},
$$

where $\Omega_{p}(0)$ and $\Omega_{n}(0)$ are the proton and neutron components of the static spin nuclear matrix elements, $\delta_{A}$ is the relative phase between them (zero or $\pi$ ) and $\delta$ the relative phase between the amplitudes $a_{p}$ and $a_{n}$.

The nuclear spin ME are defined as follows:

$$
\Omega_{p}(0)=\sqrt{\frac{J+1}{J}} \prec J J\left|\sigma_{z}(p)\right| J J \succ \quad, \quad \Omega_{n}(0)=\sqrt{\frac{J+1}{J}} \prec J J\left|\sigma_{z}(n)\right| J \quad J \succ
$$

where $J$ is the total angular momentum of the nucleus and $\sigma_{z}=2 S_{z}$. The spin operator is defined by $S_{z}(p)=$ $\sum_{i=1}^{Z} S_{z}(i)$, i.e. a sum over all protons in the nucleus, and $S_{z}(n)=\sum_{i=1}^{N} S_{z}(i)$, i.e. a sum over all neutrons. Furthermore

$$
\Omega_{0}(0)=\Omega_{p}(0)+\Omega_{n}(0), \quad \Omega_{1}(0)=\Omega_{p}(0)-\Omega_{n}(0) .
$$

The spin ME can be obtained in the context of a given nuclear model. Some such matrix elements of interest to the planned experiments are given in table [. The shown results are obtained from DIVARI [29], Ressel et al $\left(^{*}\right)$ [30], the Finish group $(* *)$ [31] and the Ioannina team $(+)$ [40], [32].

Before concluding this section we should emphasize that from the spin matrix elements of Table 1 those associated with ${ }^{19} \mathrm{~F}$ are the most reliable for the following reasons [29]:

- The light s-d nuclei are very well described within the interacting shell model.

- The magnetic moment of the ground state is dominated by the spin (the orbital part is negligible).

- The calculated magnetic moment is quite large and in good agreement with experiment.

To summarize: The proton and neutron spin cross sections can be obtained in a given particle model for the WIMP's. As we have seen there is a plethora of such models to motivate the experiments. Some of them may yield as high as a few tens of events per $\mathrm{kg}$ of target per year [12]. But most of them depend on imput parameters that are not well detemined. So none of them seems to be universally accepted. Thus in the present work, rather than following the standard procedure of providing constrained parameter spaces, we will treat the proton and neutron cross sections as parameters to be extracted from the data. This can be done, once the nuclear spin matrix elements are known, for various values of the phase difference $\delta$. The only particle parameter we will retain is the WIMP mass, which is the most important, since it enters not only in the elementary cross sections but the kinematics as well. 

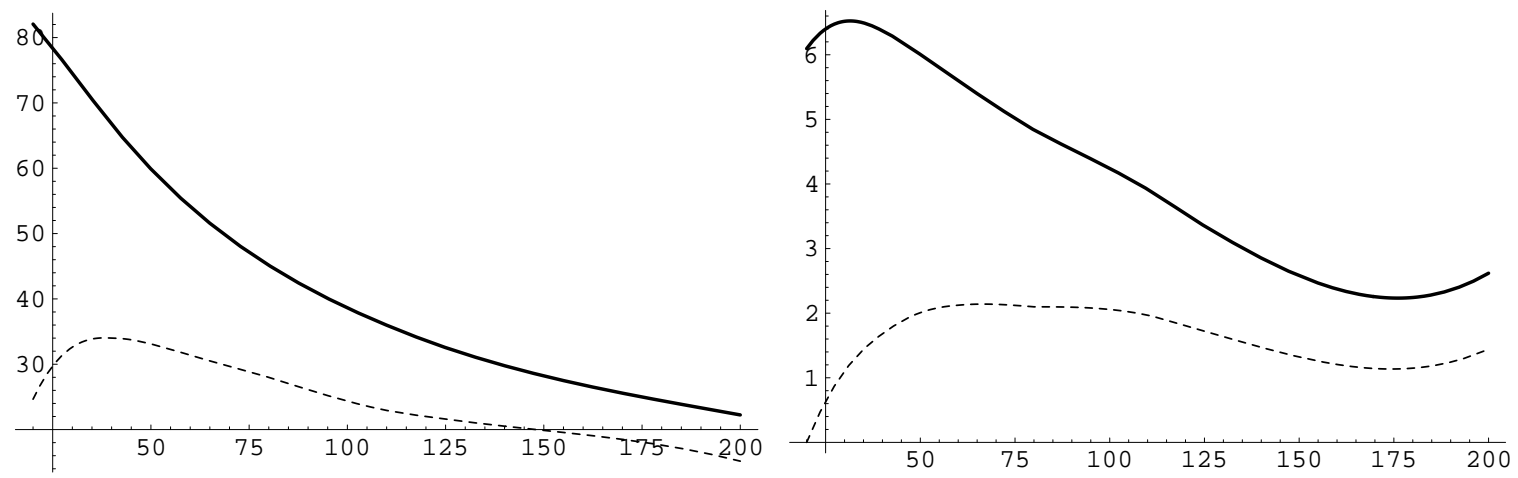

FIG. 2: On the left the quantity $c_{\text {spin }}\left(A, \mu_{r}(A), m_{\chi^{0}}\right) \zeta_{\text {spin }}$ for the $\mathrm{A}=19$ system is shown for two cut off values $Q_{\text {min }}=0$, continuous curve, and $Q_{\text {min }}=10 \mathrm{keV}$, dotted curve. On the right the same quantity is shown for the $\mathrm{A}=127$ system. The advantages of the lighter target, especially for light WIMP, are obvious.

\section{EXPRESSIONS FOR THE RATES AND SOME RESULTS}

To obtain the total rates one must fold the diffrential rate of Eq. (1) with WIMP velocity and then integrate the resultin expression over the energy transfer from $Q_{\text {min }}$ determined by the detector energy cutoff to $Q_{\max }$ determined by the maximum WIMP velocity (escape velocity, put in by hand in the M-B distribution), i.e. $v_{e s c}=2.84 v_{0}$ with $v_{0}$ the velocity of the sun around the center of the galaxy $(229 \mathrm{Km} / \mathrm{s})$.

Ignoring the motion of the Earth the total (non directional) rate is given by

$$
R=\bar{R} t\left(a, Q_{m i n}\right) \quad, \quad \bar{R}=\frac{\rho(0)}{m_{\chi^{0}}} \frac{m}{A m_{p}}\left(\frac{\mu_{r}}{\mu_{r}(p)}\right)^{2} \sqrt{\left\langle v^{2}\right\rangle}\left[\sigma_{p, \chi^{0}}^{S} A^{2}+\sigma_{n u c}^{s p i n}\right] .
$$

The WIMP parameters have been absorbed in $\bar{R}$. The parameter $t$ takes care of the nuclear form factor and the folding with WIMP velocity distribution [12, 38, 42] (for its values see table II). It depends on $Q_{\text {min }}$, i.e. the energy transfer cutoff imposed by the detector and $a=\left[\mu_{r} b v_{0} \sqrt{2}\right]^{-1}$.

In the present work we find it convenient to re-write it as:

$$
R=\tilde{K}\left(\sigma_{1}\right)\left[c_{c o h}\left(A, \mu_{r}(A), m_{\chi^{0}}\right) \frac{\sigma_{p, \chi^{0}}^{S}}{\sigma_{1}}+c_{s p i n}\left(A, \mu_{r}(A), m_{\chi^{0}}\right) \frac{\sigma_{n u c}^{\text {spin }}}{\sigma_{1}}\right]
$$

For the spin cross section it is convenient to take $\sigma_{1}=10^{-5} \mathrm{pb}$. Thus

$$
\tilde{K}\left(\sigma_{1}\right)=\frac{\rho(0)}{100 \mathrm{GeV}} \frac{m}{m_{p}} \sqrt{\left\langle v^{2}\right\rangle} \sigma_{1} \simeq 1.6010^{-2} y^{-1} \frac{\rho(0)}{0.3 G e V c m^{-3}} \frac{m}{1 K g} \frac{\sqrt{\left\langle v^{2}\right\rangle}}{280 k m s^{-1}}
$$

For the coherent mode it may be more convenient to pick $\sigma_{1}=10^{-7} p b$, which is close to the present experimental limit. Furthermore

$$
\left.\left.c_{c o h}\left(A, \mu_{r}(A), m_{\chi^{0}}\right)\right)=\frac{100 \mathrm{GeV}}{m_{\chi^{0}}}\left[\frac{\mu_{r}(A)}{\mu_{r}(p)}\right]^{2} A t_{c o h}(A), c_{s p i n}\left(A, \mu_{r}(A), m_{\chi^{0}}\right)\right)=\frac{100 G e V}{m_{\chi^{0}}}\left[\frac{\mu_{r}(A)}{\mu_{r}(p)}\right]^{2} \frac{t_{\text {spin }}(A)}{A} .
$$

The parameters $c_{c o h}\left(A, \mu_{r}(A), m_{\chi^{0}}\right), c_{s p i n}\left(A, \mu_{r}(A), m_{\chi^{0}}\right)$, which give the relative merit for the coherent and the spin contributions in the case of a nuclear target compared to those of the proton, are tabulated in table II for energy cutoff $Q_{\min }=0,10 \mathrm{keV}$. Thus via Eq. (17) we can extract the nucleon cross section from the data.

The quantity $c_{\text {spin }}\left(A, \mu_{r}(A), m_{\chi^{0}}\right) \zeta_{\text {spin }}$, when the isoscalar contribution is neglected and employing $\Omega_{1}^{2}=1.22(2.81)$ for ${ }^{127} I\left({ }^{19} \mathrm{~F}\right)$, is shown in Fig 2, In the case of the spin induced cross section, the light nucleus ${ }^{19} \mathrm{~F}$ has certainly an advantage over the heavier nucleus ${ }^{127} \mathrm{I}$ (see Fig. 2). For the coherent process, however, the light nucleus is disfavored. (see Table II). 
TABLE II: The factors $c 19=c_{c o h}\left(19, \mu_{r}(19), m_{\chi^{0}}\right), s 19=c_{s p i n}\left(19, \mu_{r}(19), m_{\chi^{0}}\right), c 19=c_{c o h}\left(73, \mu_{r}(73), m_{\chi}\right)$, $s 73=c_{\text {spin }}\left(73, \mu_{r}(73), m_{\chi}\right)$ and $c 127=c_{\text {coh }}\left(127, \mu_{r}(127), m_{\chi^{0}}\right), s 127=c_{s p i n}\left(127, \mu_{r}(127), m_{\chi^{0}}\right)$ for two values of $Q_{\text {min }}$. Also given are the factors $s 3=c_{s p i n}\left(3, \mu_{r}(3), m_{\chi}\right)$ for $Q_{\text {min }}=0$.

\begin{tabular}{|c|c|c|c|c|c|c|c|c|c|}
\hline $2 \min$ & & \multicolumn{8}{|c|}{$m_{\chi}(\mathrm{GeV})$} \\
\hline $\mathrm{keV}$ & & 20 & 30 & 40 & 50 & 60 & 80 & 100 & 20 \\
\hline 0 & $\mathrm{t}(3, \mathrm{~s})$ & 1.166 & 1.166 & 1.166 & 1.166 & 1.166 & 1.166 & 1.166 & 1.166 \\
\hline 0 & $\mathrm{c3}$ & 131 & 92.6 & 71.6 & 58.3 & 49.2 & 37.5 & 30.3 & 15. \\
\hline 0 & s3 & 14.6 & 10.3 & 7.95 & 6.48 & 5.47 & 4.16 & 3.36 & 17 \\
\hline 0 & $\bar{t}(19, c)$ & 1.153 & 1.145 & 1.138 & 1.134 & 1.130 & 1.124 & 1.121 & 1.11 \\
\hline 0 & $t(19, s)$ & 1.132 & 1.117 & 1.105 & 1.096 & 1.089 & 1.079 & 1.072 & 1.05 \\
\hline 0 & $\overline{c 19}$ & 11500 & 10500 & 9420 & 8500 & 7700 & 6450 & $\overline{5540}$ & $\overline{32}$ \\
\hline 0 & s19 & 31.2 & 28.3 & 25.4 & 22.8 & 20.6 & 17.2 & 14.6 & 8.4 \\
\hline 0 & $\frac{\mathrm{t}(23, \mathrm{c})}{\mathrm{s}}$ & 1.107 & 1.099 & 1.092 & 1.089 & 1.085 & 1.079 & 1.076 & 1.06 \\
\hline 0 & $\mathrm{t}(23, \mathrm{~s})$ & 1.075 & 1.061 & 1.050 & 1.041 & 1.035 & 1.025 & 1.018 & 1.0 \\
\hline 0 & $\mathrm{c} 23$ & $\overline{16100}$ & 15200 & 14100 & 1300 & 11900 & 10200 & 8830 & 528 \\
\hline 0 & $\mathrm{~s} 23$ & 29.5 & 27.8 & 25.6 & 23.4 & 21.4 & 18.2 & 13.8 & 9. \\
\hline 0 & $\mathrm{t}(73, \mathrm{c})$ & 1.119 & 1.083 & 1.047 & 1.014 & 0.984 & 0.933 & 0.893 & 0.78 \\
\hline 0 & $\mathrm{t}(73, \mathrm{~s})$ & 1.135 & 1.112 & 1.088 & 1.064 & 1.043 & 1.006 & 0.976 & 0.88 \\
\hline$\overline{0}$ & c73 & 13000 & 31000 & 139000 & 143000 & 42000 & $\overline{87000}$ & 30000 & 3500 \\
\hline 0 & s73 & 20.8 & 23.9 & 25.2 & 25.5 & 25.2 & 23.9 & 22.2 & 15 \\
\hline 0 & $\mathrm{t}(127, \mathrm{c})$ & 0.984 & 0.844 & 0.721 & 0.621 & 542 & 0.430 & 358 & 0.21 \\
\hline 0 & $\mathrm{t}(127, \mathrm{~s})$ & 0.948 & 0.796 & 0.671 & 0.574 & 0.501 & 0.401 & 0.340 & 0.22 \\
\hline 0 & c127 & 206000 & 225000 & 223000 & 11000 & 97000 & 69000 & 145000 & 8240 \\
\hline 0 & s127 & 12.3 & 13.1 & 12.8 & 2.1 & 11.3 & 9.7 & 8.5 & 5 \\
\hline 10 & $\bar{t}(19, c)$ & $\overline{0.352}$ & 0.511 & 0.592 & 0.639 & 0.667 & 0.710 & 0.720 & 0.77 \\
\hline 10 & $t(19, s)$ & 340 & 0.489 & 0.563 & 0.606 & 0.631 & 0.669 & 0.676 & 0.72 \\
\hline 10 & c19 & $\overline{3500}$ & 4676 & 4902 & 4789 & 4546 & 4075 & 3557 & 223 \\
\hline 10 & s19 & 9.3 & 12.4 & 12.9 & 12.6 & 11.9 & 10.6 & 9.3 & 5 \\
\hline 10 & $\mathrm{t}(73, \mathrm{c})$ & $\overline{0}$ & 0.020 & 119 & 246 & 363 & 0.539 & 0.651 & $\overline{0.84}$ \\
\hline 10 & $\mathrm{t}(73, \mathrm{~s})$ & 0 & 0.0175 & 0.105 & 0.213 & 0.311 & 0.453 & 0.539 & 0.67 \\
\hline 10 & c73) & 0 & 2310 & 15300 & 32900 & 49600 & 73400 & 86300 & 893 \\
\hline 10 & s73 & 0 & 0.39 & 2.5 & 5.3 & 7.9 & 11.6 & 13.4 & 13 \\
\hline 10 & $\mathrm{t}(127, \mathrm{c})$ & 0.000 & 56 & 205 & 0.222 & 0.216 & 191 & .175 & 0.1 \\
\hline 10 & $\mathrm{t}(127, \mathrm{~s})$ & 0.000 & 135 & 0.177 & 0.192 & 0.190 & 0.174 & 0.165 & 0.12 \\
\hline 10 & $\begin{array}{l}\mathrm{c} 127 \\
\end{array}$ & $\overline{0}$ & $\overline{1500}$ & 200 & $\overline{5500}$ & $\overline{8500}$ & 4900 & 71000 & $\overline{4220}$ \\
\hline 10 & s127 & 0. & 2.2 & 3.4 & 4.0 & 4.3 & 4.2 & 4.1 & \\
\hline
\end{tabular}

TABLE III: The experimental sensitivity ratios (ESR) for various targets assuming a WIMP mass of 50 GeV. $p, n$ and $i v$ correspond to the elementary proton, neutron and isovector dominance respectively.

\begin{tabular}{||r|r|r|r|r|r||}
\hline \hline & ${ }^{3} \mathrm{He}$ & ${ }^{19} \mathrm{~F}$ & ${ }^{23} \mathrm{Na}$ & ${ }^{73} \mathrm{Ge}$ & ${ }^{127} \mathrm{I}$ \\
\hline$p$ & $1.4 \times 10^{3}$ & $4.1 \times 10^{2}$ & $2.7 \times 10^{3}$ & $1.3 \times 10^{7}$ & $2.5 \times 10^{4}$ \\
\hline$n$ & $1.4 \times 10$ & $1.2 \times 10^{6}$ & $4.3 \times 10^{5}$ & $1.6 \times 10^{4}$ & $4.2 \times 10^{5}$ \\
\hline $\mathrm{iv}$ & $1.1 \times 10$ & $4.0 \times 10^{2}$ & $3.2 \times 10^{3}$ & $1.7 \times 10^{4}$ & $4.3 \times 10^{4}$ \\
\hline \hline
\end{tabular}

The experimental sensitivity ratios (ESR), i.e. the extracted from experiment nucleon cross section ratios satisfy:

$$
E S R=\frac{\sigma_{k, \chi^{0}}^{\text {spin }}}{\sigma_{p, \chi^{0}}^{S}}=\left[\frac{c_{c o h}\left(A, \mu_{r}(A), m_{\chi^{0}}\right)}{c_{\text {spin }}\left(A, \mu_{r}(A), m_{\chi^{0}}\right)}\right] \frac{3}{\Omega_{k}^{2}}, k=p, n, i v, \text { for proton, neutrom, isovector respectively }
$$

The quantity ESR for a WIMP mass of $50 \mathrm{GeV}$ is shown in table III. It is clear from this table why the limits on the spin cross section extracted from all targets is much bigger compared to that extracted for the coherent mode. 
We should emphasize that the elementary cross sections do not depend on the target. It is only the values extracted from experiment that do so, giving a measure of the sensitivity of the various experiments. The elementary cross sections only depend on the particle model and the structure of the nucleon. Thus, e.g., in the case of K-K WIMPs the coherent cross section dominates, if the WIMP is a K-K gauge boson, but the spin cross section is bigger, when the WIMP is a K-K neutrino [16].

If the effects of the motion of the Earth around the sun are included, the total non directional rate is given by

$$
\begin{aligned}
& R=\tilde{K}\left(\sigma_{1}\right)\left[c_{\operatorname{coh}}\left(A, \mu_{r}(A), m_{\chi^{0}}\right) \frac{\sigma_{p, \chi^{0}}^{S}}{\sigma_{1}}\left(1+h\left(a, Q_{\text {min }}\right) \cos \alpha\right)\right] \text { (coherent), } \\
& R=\tilde{K}\left(\sigma_{1}\right)\left[c_{\text {spin }}\left(A, \mu_{r}(A), m_{\chi^{0}}\right) \frac{\sigma_{n u c}^{s p i n}}{\sigma_{1}}\left(1+h_{\text {spin }}\left(a, Q_{\text {min }}\right) \cos \alpha\right)\right] \text { (spin), }
\end{aligned}
$$

where $h\left(h_{\text {spin }}\right)$ are the modulation amplitudes and $\alpha$ is the phase of the Earth, which is zero around June 2 nd. We are going to only briefly discuss the modulation amplitudes here since they depend only on the WIMP mass and are independent of the other particle parameters. In the case of the two very light targets, however, they are pretty independent of the WIMP mass. In fact for the light systems :

$$
h=h_{\text {spin }}=0.0232\left(\text { for } \mathrm{A}=3 \text { ) and } h=0.0229, h_{\text {spin }}=0.0227 \text { (for } \mathrm{A}=19\right) .
$$

Actually for the $\mathrm{A}=19$ system there is about (10\%) reduction as the WIMP mass increases.

In the case of the target ${ }^{3} \mathrm{He}$ the quantity $t$ is also essentially independent of the WIMP mass, since the WIMP is expected to be much heavier than the nuclear mass. From table $\amalg$ we see that the coherent rate is quite small for this light system, but the spin induced rate is only a factor of two smaller than that for ${ }^{19} \mathrm{~F}$. As we have already mentioned this nucleus, has definite experimental advantages [41].

In many instances the experiments are interested in the differential event rate. This is a function of two variables, the WIMP mass and the energy transfer $Q$. For the light systems, however, the dependence on the WIMP mass is rather weak, especially for heavy WIMPs. Thus the presentation of the results is relatively simple and we are going to present them here. One finds:

$$
\frac{d R}{d Q}=\tilde{K}\left(\sigma_{1}\right)\left[\frac{d R_{0}\left(Q, A, m_{\chi}\right)}{d Q}\left(1+H\left(Q, A, m_{\chi}\right) \cos \alpha\right) \frac{\sigma_{n u c}^{\text {spin }}}{\sigma_{1}}\right],
$$

with an analogous expression for the coherent mode. The time average quantity $d R_{0}\left(Q, A, m_{\chi}\right) / d Q$ and the relative modulation amplitude $H\left(Q, A, m_{\chi}\right)$ are shown in Fig. 3. The differential cross section is normalized so that the area under the corresponding curve gives the value $c_{\text {spin }}\left(A, \mu_{r}(A), m_{\chi^{0}}\right)$ of Eq. (22). Note that the quantity $\mathrm{H}$, being the ratio of two amplitudes, the amplitude for modulation divided by the time independent amplitude, is independent of the nuclear model. So it is the same for the spin and coherent mode. Note also that, at relatively low energy transfers, $H$ becomes negative, i.e. minimum in June and maximum in December. The negative value, however, for the light targets is small for all WIMP masses. For this reason for a light target the integrated modulation amplitude $h$ is always positive (maximum in June, minimum in December, as expected). The modulation curves $H$ keep increasing as the energy transfer increases, mainly because the time independent amplitude, coming in the denominator, decreases. Thus in spite of this increase of $H, h$ remains constant.

\section{RESULTS FOR THE SPIN CONTRIBUTION}

\section{One amplitude is dominant}

This occurs in cases when the nuclear structure leads to a dominant spin ME, like ${ }^{19} \mathrm{~F}$ with a dominant proton component. In this case, barring unusual circumstances at the quark level favoring the component not favored by nuclear physics, the analysis is simple. Thus, e.g., in the case of ${ }^{19} \mathrm{~F}\left(\Omega_{p}=1.645, \Omega_{n}=-0.030\right.$ the event rate for an elementary cross section of $10^{-5} \mathrm{pb}$ is exhibited as a function of the WIMP mass in Fig. 4. From these plots, for a given WIMP mass, one may extract limits on the relevant nucleon cross section from the experimental limits. Using the event rate of $13.75 \mathrm{Kg}-\mathrm{d}$ or $5020 \mathrm{Kg}-\mathrm{y}$ of PICASSO 24] and the most favorable WIMP mass of $30 \mathrm{GeV}$, from Fig. 4 we extract a proton spin cross section of $0.1 \mathrm{pb}$, to be compared with the value of $0.16 \mathrm{pb}$ extracted there 24]. From Eq. (20) we extract a coherent cross section of $2.5 \times 10^{-4} \mathrm{pb}$ for this system, which is poor compared to the limits of CDMS [6] and XENON [7]. The PICASSO people are fighting with their new detector against the $\alpha$ background, with a flat plateau in the region of their signal, and their limit will soon substantially improve . 


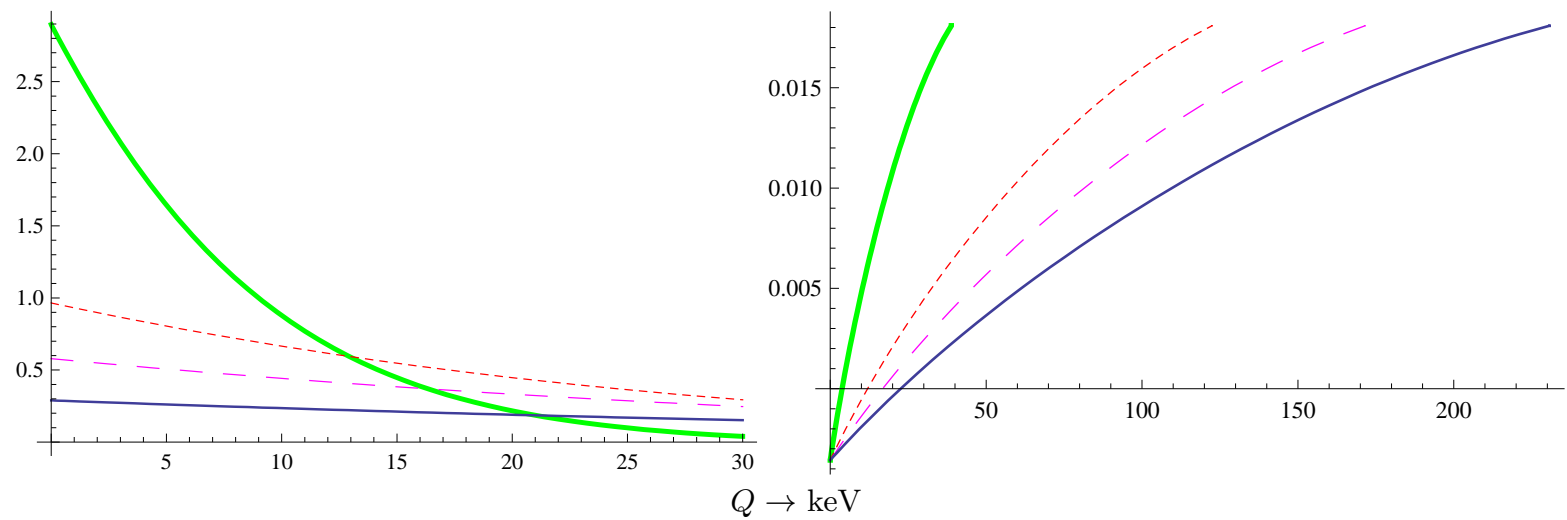

FIG. 3: On the left the quantity $\frac{d R_{0}\left(Q, A, m_{\chi}\right)}{d Q}$ and on the right the quantity $H\left(Q, A, m_{\chi}\right.$ involving the spin induced process for the $\mathrm{A}=19$ system as a function of the energy transfer $Q$ in $\mathrm{keV}$. The thick solid, the dotted, the dashed and the thin solid lines correspond to WIMP masses 10,30, 50 and $100 \mathrm{GeV}$ respectively. On the left panel the range

of $Q$ is restricted to make the curve for low WIMP mass more visible. For masses heavier than $30 \mathrm{GeV}$ the differential event rate has essentially a constant slope. So it is adequate to restrict ourselves to low $Q$. The full range of Q can be inferred from the right panel.
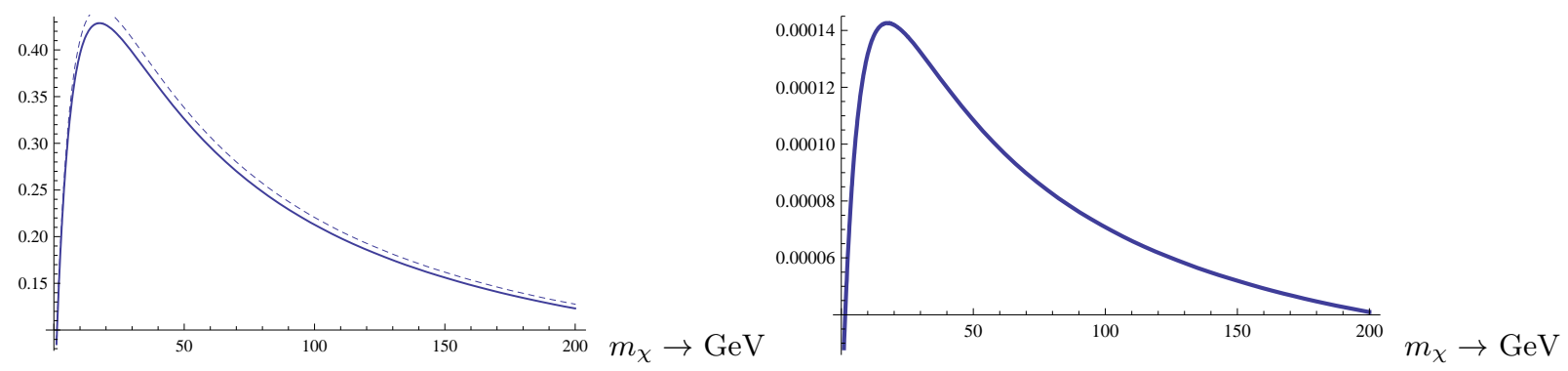

FIG. 4: The event rate (kg-y) for the target ${ }^{19} \mathrm{~F}$ assuming a nucleon cross section of $10^{-5} \mathrm{pb}$ as a function of the WIMP mass in GeV. In the left panel the continuous curve takes into account only the proton component. The dotted curve results when the proton and neutron cross sections are the same, but the corresponding amplitudes are opposite (the isoscalar amplitude is assumed to vanish). The difference is small. In the right panel we consider the

case that the elementary proton cross section vanishes. In this case the nuclear structure suppresses the rate.

\section{Exclusion plots in the $\sqrt{\sigma_{p}}, \sqrt{\sigma_{n}}$ plane}

From the experimental data, using the nuclear spin matrix elements,one can extract a restricted region in the $\sigma_{p}, \sigma_{n}$ plane [43]-[44]. The relevant relation is:

$$
|| \Omega_{p}\left|\sqrt{\sigma_{p}}+\right| \Omega_{n}\left|\sqrt{\sigma_{n}} e^{i\left(\delta+\delta_{A}\right)}\right|^{2}=\sigma_{1} \frac{3 R}{\tilde{K}\left(\sigma_{1}\right) s A} .
$$

where $s A$ is a short hand notation for $c_{\text {spin }}\left(A, \mu_{r}(A), m_{\chi^{0}}\right)$. The extracted values, given the event rate and the spin $\mathrm{ME}$, depend on the WIMP mass and the relative phase of the two amplitudes.

Since the procedure is much more complicated than that entering the analysis of the coherent node, a few explanations regarding the presentation of our results (Figs [5] [10) are in order:

- We found it more convenient to present in the plots the extracted $\sqrt{\sigma_{p}}$ and $\sqrt{\sigma_{n}}$ rather than the cross sections themselves.

- For illustrative purposes the dependence on $\delta$ can be given in a simple graph whereby the cross sections can be expressed in units containing all the parameters. The extracted shapes, which depend on $\delta_{A}$ are shown in Fig. 5. Such a plot, in principle, contains all the needed information, but it is too general to be practical. 


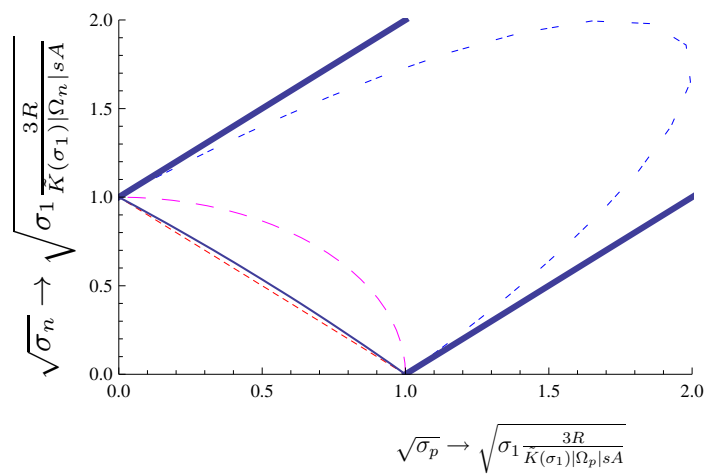

(a)

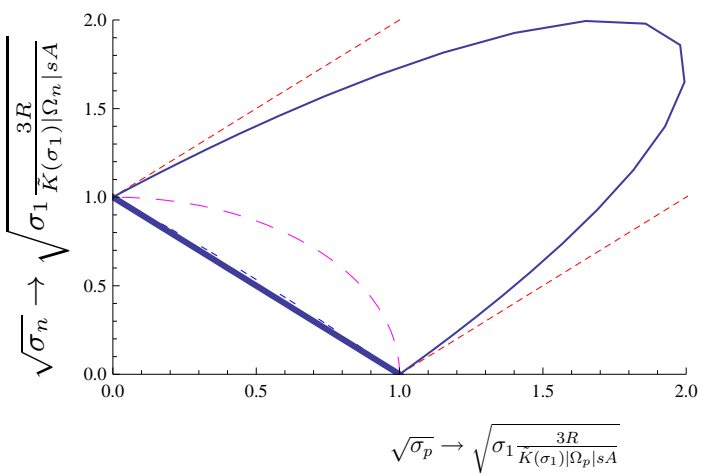

(b)

FIG. 5: A "universal" exclusion plot in the $\left(\sqrt{\sigma_{p}}, \sqrt{\sigma_{n}}\right)$ plane exhibiting the dependence on the phase $\delta$. On te right panel the nuclear spins are of the same sign, while on the other of opposite signs. When the two amplitudes are relatively real, they are not bounded except when the the phase $\delta$ is the same with the relative phase of the two nuclear matrix elements. In both panels the dotted, the fine solid, the dashed, the dotted- dashed, and the thick solid curve correspond to $\delta=0, \pi / 6, \pi / 2,5 \pi / 6$ and $\pi$ respectively.

- The contour for $\delta \neq 0, \pi$ is in general an ellipse. For a given experimental bound, the allowed values of the cross sections are in the space enclosed by an ellipse. One can see that, depending on $\delta$ the maximum allowed cross sections can be quite a bit higher than those extracted assuming a single mode. If the two amplitudes are relatively real, then the contours become straight lines and the cross sections may be constrained, but only if the relative phase of the two amplitudes is the same with $\delta_{A}$. If they differ by $\pi$, the individual cross sections are not bounded, they can be anywhere between the two lines.

- For given nuclear spin ME the extracted $\sqrt{\sigma_{p}}$ and $\sqrt{\sigma_{n}}$ are presented in units $\sqrt{\sigma_{1} \frac{3 R}{\left.K\left(\sigma_{1}\right) s A\right)}}$ (see Fig. [6-10 ). Once the experiment determines the rate $R$ and the parameter $s A$, for the chosen WIMP mass, is read off from table II one can immediately extract from the figures the cross sections in units of $\sigma_{1}\left(\tilde{K}\left(\sigma_{1}\right)\right.$ is given by Eq. (18)). As an illustration we do this on the right panel of the the figures $6-10$ assuming an event rate of 1 event per $\mathrm{Kg}$ target per year for the optimum value of $m_{\chi}$ (maximum of $s A$ )

The following cases are of experimental interest:

i) We first consider the case of nuclear spin matrix elements of opposite sign and $\left|\Omega_{n}\right|>\left|\Omega_{p}\right|$ as is the case of the $\mathrm{A}=3$ system. The exclusion plots are shown in Fig. 6]

ii) Next comes the case of spin matrix elements of opposite sign and $\left|\Omega_{p}\right| \gg\left|\Omega_{n}\right|$ as is the case of the ${ }^{19} \mathrm{~F}$ target. This case has already been analyzed above, considering only protons. Just in case the elementary proton cross section is very suppressed, we present the relevant exclusion plots in Fig. 7

iii) After this we consider nuclear spin matrix elements of same sign and $\left|\Omega_{n}\right| \gg\left|\Omega_{p}\right|$. This is the case of the ${ }^{73} \mathrm{Ge}$ target. The relevant exclusion plots are shown in Fig. 8

iv We consider the case with both spin matrix elements being significant. Such may be the case of the ${ }^{127}$ I target $\left(\Omega_{p}=1.127, \Omega_{n}=0.315\right)$. The resulting exclusion plots are shown in Fig. 9 ,

v) We finally show the exclusion plots exhibited by another proton favoring nucleus, ${ }^{23} \mathrm{Na}$, which is present together with ${ }^{127} \mathrm{I}$ in the target NaI (see Fig. 10). We notice that, since one has the same number of nuclei of each component in a given mass of the target, ${ }^{23} \mathrm{Na}$ competes well with the ${ }^{127} \mathrm{I}$ in the spin induced event rate.

\section{CONCLUDING REMARKS}

We have analyzed the spin induced WIMP nucleus elastic cross section and related event rates. Both depend rather sensitively on the spin structure of the nucleus. Barring unusual circumstances at the elementary level, the spin mode has no chance to compete with the coherent WIMP nucleus scattering in the case of heavy targets. It could, however, compete with it in the case of light targets. For light targets, and in particular for ${ }^{3} \mathrm{He}$ and ${ }^{19} \mathrm{~F}$, we believe the nuclear matrix elements are very accurate to allow reliable extraction of the nucleon cross sections from the data, if and when they become available. In the cases considered here, with the possible exception of ${ }^{127} \mathrm{I}$, 


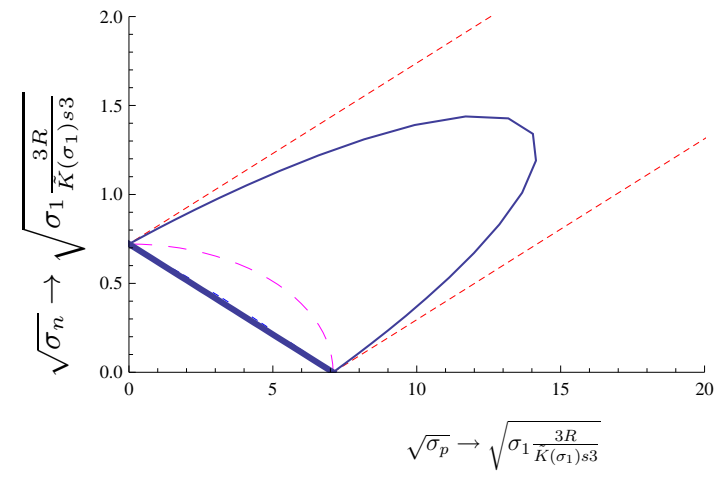

(a)

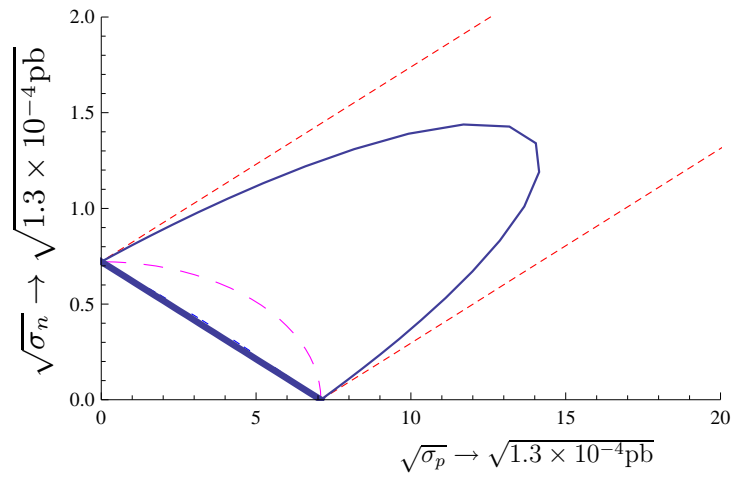

(b)

FIG. 6: The exclusion plot in the $\left(\sqrt{\sigma_{p}}, \sqrt{\sigma_{n}}\right)$ plane in the case of the target ${ }^{3} \mathrm{He}$ for various values of the phase $\delta$ using the relevant spin ME of table I. The units depend on the parameters of table II, the experimental rate $R$ as well as and, for the chosen scale of $\sigma_{1}$, on $\tilde{K}\left(\sigma_{1}\right)$ (a). The same exclusion plot in the case of a WIMP with a mass 20 $\mathrm{GeV}$ normalized to 1 event per $\mathrm{kg}$ target per year in the indicated units, obtained using $\tilde{K}\left(\sigma_{1}\right)=1.6 \times 10^{2} \mathrm{y}^{-1}$ and $\sigma_{1}=10^{-5} \mathrm{pb}(\mathrm{b})$. Cross sections for other event rates can be trivially extracted by a simple rescaling of panel (b).

When the two amplitudes are relatively real $(\delta=0, \pi)$, they are not bounded except when $\delta$ coincides with the phase difference $\delta_{A}$ of the neclear matrix elements. For the labelling of the curves see Fig: 5 .

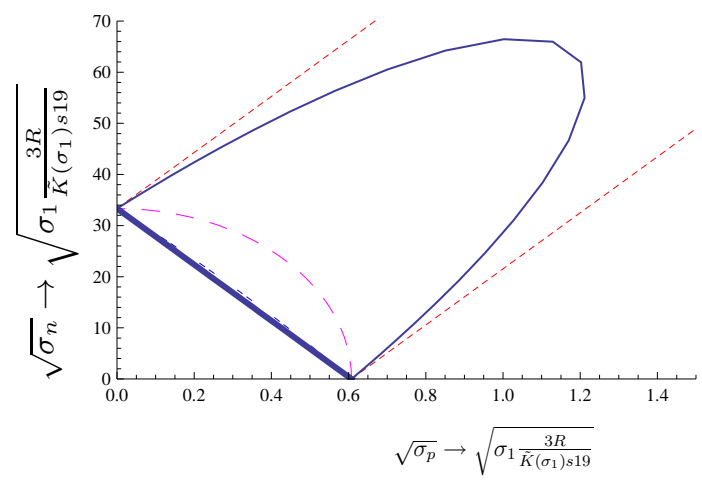

(a)

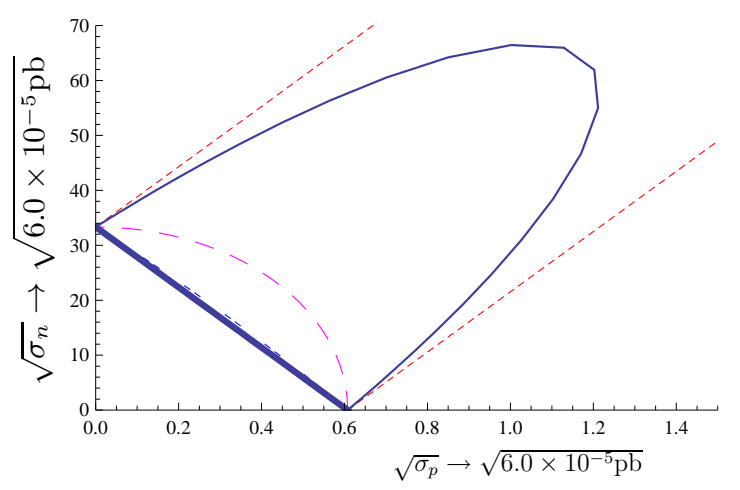

(b)

FIG. 7: The same as in Fig. 6 in the case of ${ }^{19} \mathrm{~F}$ target.

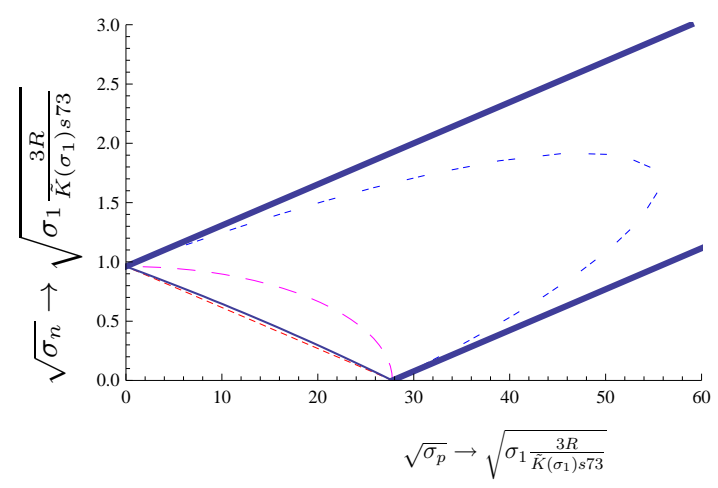

(a)

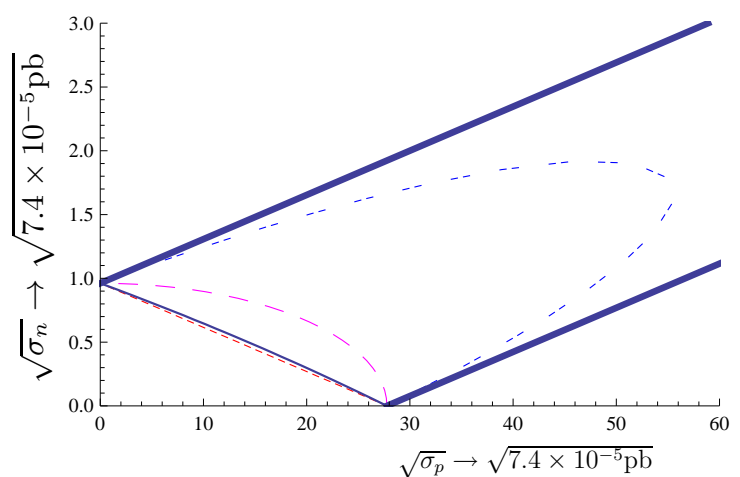

(b)

FIG. 8: The same as in Fig. 6 in the case of ${ }^{73}$ Ge target. Now in panel (b) we exhibit the most sensitive case of a WIMP mass of $50 \mathrm{GeV}$. 


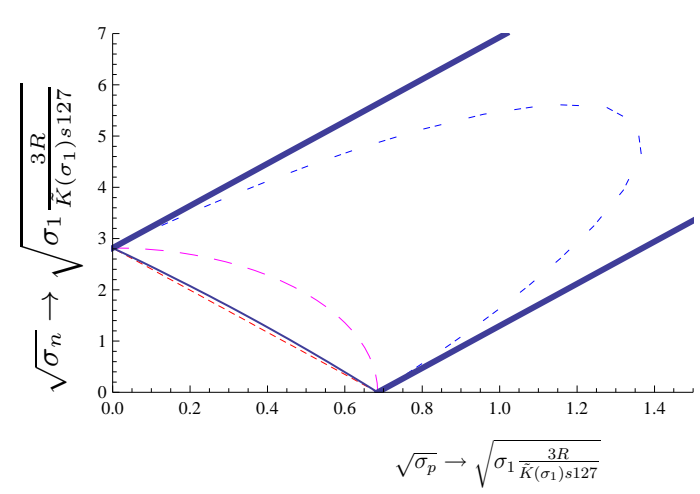

(a)

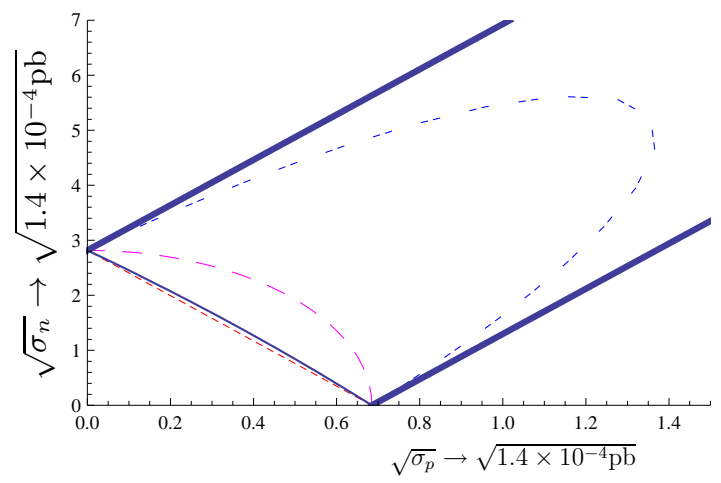

(b)

FIG. 9: The same as in Fig. [6 in the case of ${ }^{127} \mathrm{I}$ target. Now in panel (b) we exhibit the most sensitive case of a WIMP mass of $30 \mathrm{GeV}$.

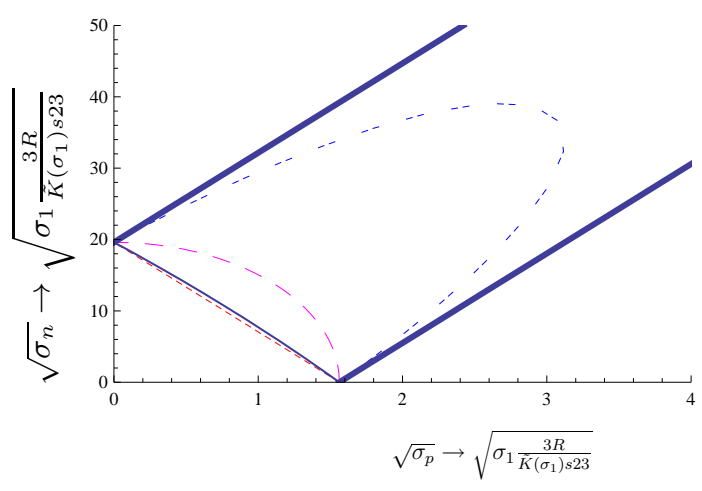

(a)

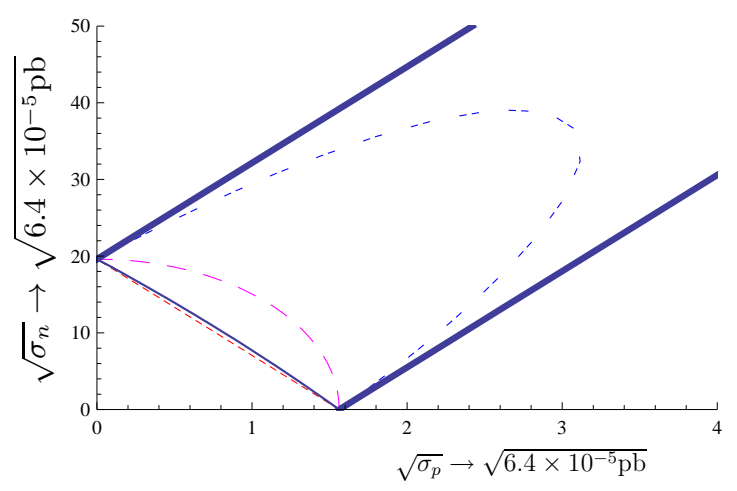

(b)

FIG. 10: The same as in Fig. 6 in the case of ${ }^{23} \mathrm{Na}$ target. Now in panel (b) we exhibit the most sensitive case of a WIMP mass of $20 \mathrm{GeV}$.

the nuclear structure tends to favour the proton or the neutron component. This allows a simple extraction of the corresponding nucleon cross section. This is also true even if both components are present, but the isoscalar amplitude at the nucleon level is suppressed. Finally, even if both the proton and the neutron amplitudes are important, we have shown that knowledge of the nuclear matrix elements allows one to draw suitable exclusion plots. Unfortunately, then, the situation is technically a bit complicated by the fact that one must draw one exclusion plot for each WIMP mass. So, for targets with spin different from zero, exclusion plots should be drawn as more experimental data become available.

\section{ACKNOWLEDGMENTS}

The final stages of this work were completed while the author visited Tuebingen under a Humboldt Research Award. The Author is indebted for this opportunity to Alexander von Humboldt Foundation and Professor Amand Faessler.

[1] E. Komatsu et al., Astrophys.J.Suppl. 180 (2009), arXiv:0803.0547(atro-ph).

[2] D. P. Bennett and et al, Phys. Rev. Lett. 74, 2867 (1995).

[3] R. Bernabei and et al, Phys. Lett. B 389, 757 (1996).

[4] R. Bernabei et al, Phys. Lett. B 424, 195 (1998). 
[5] A. Benoit et al, [EDELWEISS collaboration]: Phys. Lett. B 545, 43 (2002);

V. Sanglar,[EDELWEISS collaboration] arXiv:astro-ph/0306233;

D. S. Akerib et al,[CDMS Collaboration]: Phys. Rev D 68, 082002 (2003); arXiv:astro-ph/0405033.

[6] J. Yoo [CDMS Collaboration], arXiv:0810.3527 [hep-ex].

[7] J. Angle and et al, Phys. Rev. Lett. 100, 021303 (2008), arXiv:0706.0039.

[8] G. Jungman, M. Kamionkowski, and K. Griest, Phys. Rep. 267, 195 (1996).

[9] A. Bottino et al., Phys. Lett B 402, 113 (1997).

R. Arnowitt. and P. Nath, Phys. Rev. Lett. 74, 4592 (1995); Phys. Rev. D 54, 2374 (1996); hep-ph/9902237.

V. A. Bednyakov, H.V. Klapdor-Kleingrothaus and S.G. Kovalenko, Phys. Lett. B 329, 5 (1994).

[10] U. Chattopadhyay and D. Roy, Phys. Rev. D 68, 033010 (2003), hep-ph/0304108.

[11] B. Murakami and J. Wells, Phys. Rev. D p. 015001 (2001), hep-ph/0011082.

[12] J. D. Vergados, J.Phys. G 30, 1127 (2004), 0406134.

[13] J. Ellis, K. A. Olive, Y. Santoso, and V. C. Spanos, Phys.Rev. D 70, 055005 (2004).

[14] J. Hisano, S. Matsumoto, M. M. Nojiri, and O. Saito, Phys.Rev. D 71, 015007 (2005).

[15] J. Ellis, K. A. Olive, Y. Santoso, and V. C. Spanos, Phys.Rev. D 71, 095007 (2005), hep-ph/0502001.

[16] V. Oikonomou, J. Vergados, and C. C. Moustakidis, Nuc. Phys. B 773, 19 (2007).

[17] C. Kouvaris, Phys. Rev. D 76, 015011 (2007), arXiv:hep-ph/0703266.

[18] S. B. Gudnason, C. Kouvaris, and F. Sannino, Phys. Rev. D 74, 095008 (2006), arXiv:hep-ph/0608055.

[19] M.Yu.Khlopov, Kouvaris, and F. Sannino, Pys. Rev. D 78, 065040 (2008), arXiv: 0806.1191 [astro-ph].

[20] T. Ryttov and F. Sannino, Phys. Rev. D 78, 115010 (2008).

[21] H. Ejiri, K. Fushimi, and H. Ohsumi, Phys. Lett. B 317, 14 (1993).

[22] J. D. Vergados, P. Quentin, and D. Strottman, IJMPE 14, 751 (2005), hep-ph/0310365.

[23] D. Santos et al, The MIMAC-He3 Collaboration, A New ${ }^{3}$ He Detector for non Baryonic Dark Matter Search, Invited talk in idm2004 (to appear in the proceedings).

[24] Dark Matter Spin-Dependent Limits for WIMP Interactions on 19-F by PICASSO Archambault, F. Aubin, M. Auger, E. Behnke, B. Beltran, K. Clark, X. Dai, A. Davour, J. Farine, R. Faust, M.-H. Genest, G. Giroux, R. Gornea, C. Krauss, S. Kumaratunga, I. Lawson, C. Leroy, L. Lessard, C. Levy, I. Levine, R. MacDonald, J.-P. Martin, P. Nadeau, A. Noble, M.-C. Piro, S. Pospisil, T. Shepherd, N. Starinski, I. Stekl, C. Storey, U. Wichoski, V. Zacek ; arXiv:0907.0307[hep-ex].

[25] J. D. Vergados, J. of Phys. G 22, 253 (1996).

[26] M. Drees and M. M. Nojiri, Phys. Rev. D 48, 3843 (1993); Phys. Rev. D 47, 4226 (1993).

[27] A. Djouadi and M. K. Drees, Phys. Lett. B 484, 183 (2000); S. Dawson, Nucl. Phys. B 359, 283 (1991); M. Spira it et al, Nucl. Phys. B453, 17 (1995).

[28] T. P. Cheng, Phys. Rev. D 38, 2869 (1988); H-Y. Cheng, Phys. Lett. B 219, 347 (1989).

[29] P. C. Divari, T. S. Kosmas, J. D. Vergados, and L. D. Skouras, Phys. Rev. C 61, 054612 (2000).

[30] M. T. Ressell et al., Phys. Rev. D 48, 5519 (1993); M.T. Ressell and D. J. Dean, Phys. Rev. C 56, 535 (1997).

[31] E. Homlund and M. Kortelainen and T. S. Kosmas and J. Suhonen and J. Toivanen, Phys. Lett B, 584,31 (2004); Phys. Atom. Nucl. 67, 1198 (2004).

[32] T. S. Kosmas and J. D. Vergados, Phys. Rev. D 55, 1752 (1997).

[33] J. Vergados, S. N. Hansen, and O. Host, Phys. Rev. D 77, 023509 (2008).

[34] J. Vergados, AJ 137, 10 (2009), arXiv:08110382/astro-ph.

[35] K. F. A. K. Drukier and D. N. Spergel, Phys. Rev. D 33, 3495 (1986).

[36] K. Frese, J. A. Friedman, and A. Gould, Phys. Rev. D 37, 3388 (1988).

[37] J. D. Vergados, Phys. Rev. D 58, 103001 (1998).

[38] J. D. Vergados, Phys. Rev. D 63, 06351 (2001).

[39] The Strange Spin of the Nucleon, J. Ellis and M. Karliner, hep-ph/9501280.

[40] J. D. Vergados, Part. Nucl. Lett. 106, 74 (2001), hep-ph/0010151.

[41] E. Moulin, F. Mayet, and D. Santos, Phys. Lett. B 614, 143 (2005).

[42] J. D. Vergados, Phys. Rev. D 62, 023519 (2000).

[43] C. Savage, P. Gondolo, and K. Freese, Phys. Rev. D 70, 123513 (2004).

[44] F. Giuliani and T. A. Girard, Phys.Lett. B 588, 151 (2004). 